\title{
A SHORT NOTE ON THE SCHUR-JACOBSON THEOREM
}

\author{
R. C. COWSIK
}

(Communicated by Louis J. Ratliff, Jr.)

\begin{abstract}
An upper bound for the length of a commutative Artinian ring in terms of the length of a faithful module is given. This generalizes a theorem of Schur and Jacobson.
\end{abstract}

Long ago, in days of yore, Schur [3] proved that commutative subalgebras of $M_{n}(\mathbf{C})$ have dimension less than or equal to $\left[n^{2} / 4\right]+1$. In 1945 Jacobson [2] proved the same inequality for any field $K$. His proof consists of putting the matrices in the subalgebra in a "standard form" by conjugation. Later many proofs by manipulation of matrices were given. In 1976 Gustafson [1] proved the theorem by representation theoretic methods. In his paper Gustafson asked whether the theorem could be proved for Artinian rings (which are not algebras) in terms of length. Here we prove the following theorem (the proof of which is essentially Gustafson's proof of his theorem) answering the question. We thank W. Brown for bringing back to mind the problem.

Theorem 1. Let $A$ be a commutative Artinian ring and let $M$ be a faithful $A$-module of length $n$. Then $l(A) \leq\left[n^{2} / 4\right]+1$. (Here $l$ denotes the length function and $[x]$ denotes the integral part of $x$.)

Proof. We may assume by going to the components of $A$ that $A$ is a local ring with maximal ideal $\mathbf{m}$.

Lemma 2. Let $M$ and $N$ be A-modules, $N$ having finite length. Then,

$$
l(\operatorname{Hom}(M, N)) \leq \mu(M) \cdot l(N) .
$$

Proof. Let $\mu(M)=m$. We have an exact sequence $A^{m} \rightarrow M \rightarrow 0$. Homing this into $N$ we get: $\operatorname{Hom}(M, N)$ is a submodule of $N^{m}$ that has length $m$. $l(N)$. So the lemma is proved.

[Aliter: This can also be proved by induction on length of $N$.]

To continue the proof of the theorem, as $M$ is a faithful $A$-module, m injects into $\operatorname{Hom}(M, \mathbf{m} M)$. Applying the lemma we get

$$
\begin{aligned}
l(\mathbf{m}) & \leq l(\operatorname{Hom}(M, \mathbf{m} M)) \leq \mu(M) \cdot l(\mathbf{m} M) \\
& =\mu(M) \cdot\{l(M)-\mu(M)\} \leq\left[(l(M))^{2} / 4\right] .
\end{aligned}
$$

Whence, $l(A)=l(\mathbf{m})+1 \leq\left[(l(M))^{2} / 4\right]+1$.

Received by the editors November 14, 1991.

1991 Mathematics Subject Classification. Primary 13E10; Secondary 16G10. 


\section{REFERENCES}

1. W. H. Gustafson, On maximal commutative algebras of linear transformations, J. Algebra 42 (1976), 557-563.

2. N. Jacobson, Schur's Theorems on commutative matrices, Bull. Amer. Math. Soc. 50 (1946), 431-436.

3. I. Schur, Zur theorie vertauschbaren matrizen, Crelle's J. 130 (1905), 66-76.

Centre for Advanced Study in Mathematics, University of Bombay, Bombay 400098 , INDIA 